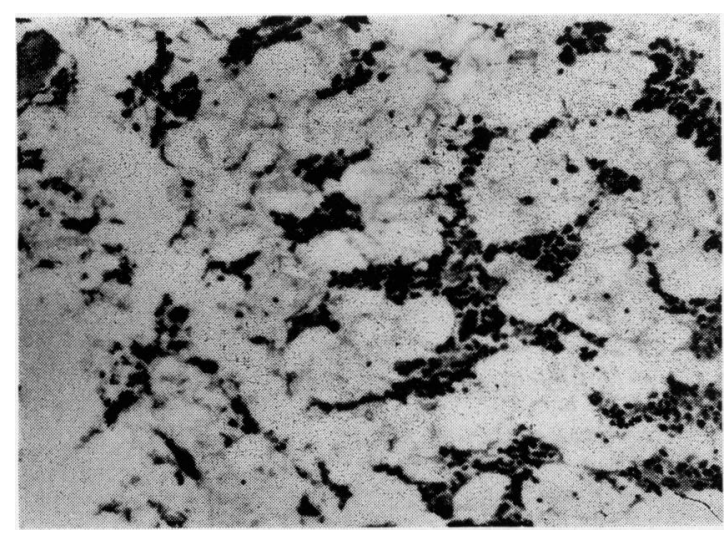

Fig. 2. A section of vertebral bone marrow from Case 2. Mucus secreting cells could not be identified even with the aid of stains for mucus. Alcian blue stain, $\times 120$.

A barium meal was difficult to perform because of his debility, and no gross abnormality was noted. His haemoglobin concentration was $12.9 \mathrm{~g} / 100 \mathrm{ml}$. Faecal occult blood was detected. His condition deteriorated rapidly, and his blood urea concentration rose. He died without further relevant investigations.

Necropsy. There were 2.6 litres of ascitic fluid. The stomach had a thickened wall especially posteriorly, caused by a mucus secreting infiltrating carcinoma. Carcinoma had spread into the peritoneum and produced adhesions which had partially obstructed the ascending colon, and produced retroperitoneal fibrosis with obstruction of the ureters.

The vertebral marrow showed no macroscopic or microscopic evidence of cancer cell metastasis there, but the fat had been replaced by mucus (Fig. 2).

\section{Discussion}

Both cases had a mucus-secreting infiltrating carcinoma of the stomach with retroperitoneal involvement. In the first case, it appears likely that the tumour had been present for 7 years, a remarkable survival for any form of therapy. The large quantity of mucus produced by the tumour deposits appears to be able to track to the marrow, and over a length of time, replace the fat deposits. Considering the gastric lesions, both patients appeared to be able to maintain their haemoglobin levels reasonably despite the marrow changes. With these tumours, gastric or oesophageal obstruction or radiological evidence of filling defects were not marked, so that the presence of mucus in the spinal or pelvic marrow may be of some diagnostic assistance in the investigation of gastric or retroperitoneal pathology.

\section{Acknowledgments}

I wish to thank the Chairman of the Repatriation Com mission for permission to publish this report, Mr W. Ruffnet for assistance with the photography, Miss $H$. Wischusen and Mr D. McKenzie for technical assistance.

\title{
Placenta praevia and ruptured exomphalos: a problem in diagnosis
}

\author{
Cyril S. Pallewela* \\ M.B., M.R.C.O.G. \\ Registrar, \\ City of London Maternity Hospital
}

THIS case is presented as a problem in diagnosis and management.

The occurrence of placenta praevia with exomphalos is extremely rare. On reviewing the literature as far back as 1885 no such case seems to have been reported. The incidence of exomphalos has been variously reported as 1 in 5000 to 1 in 11,000 live births and that of placenta praevia alone 1 in 200 . The incidence of foetal abnormality associated with placenta praevia is quoted by Macafee as $3.4 \%$. This, however, takes the form of spina bifida, hydrocephalus and anencephalus. As stated earlier, the incidence of a combination has not been worked out.

\footnotetext{
* Present address: St Albans City Hospital.
}

\section{Case report}

A young girl, Miss J.A., aged 20 years, unmarried, booked for her first pregnancy on 8 May 1967, following a period of amenorrhoea of 15 weeks.

On examination her general condition was satisfactory. The size of the uterus corresponded to dates.

Investigations: WR and GCFT negative; group $O$ Rhesus positive; haemoglobin, $81 \%$; blood pressure, 100/60; cardiovascular and respiratory systems, normal.

From about 30 weeks onwards the height of the fundus was noted to be smaller than the dates suggested. At the 35th week by dates the foetus was 
found to be a breech presentation, the height of the fundus being about 30 weeks, and an external cephalic version attempted at this time failed. When she was 36 weeks by dates she was admitted to hospital because of a history of painless haemorage from the genital tract.

On examination the height of the fundus was about 32 weeks, breech presenting; foetal heart sounds heard clearly; foetal parts easily felt. There was no area of tenderness in the uterus. A tentative diagnosis of placenta praevia was made and expectant treatment carried out. Two days later she had a further small bleed which did not warrant any interference. On the next day she had a fairly brisk haemorrhage. On abdominal examination at this stage the head was felt under the right subcostal margin. The lower segment felt very soft and boggy. The foetal heart was well heard.

The diagnosis was thought to be an anterior placenta praevia and as she was bleeding it was decided to perform an examination under anaesthesia with preparations for a Caesarean section if necessary.

Findings at operation: many clots in the vagina. The cervix was taken up. The os was two fingers dilated. A soft, boggy mass was felt occupying the lower uterine segment. The mass was felt to be in two halves.

The diagnosis considered was placenta praevia or blood clot; the consistency did not fit in with either diagnosis.

As she was bleeding fairly briskly it was decided to perform Caesarean section.

On opening the peritoneal cavity, the lower segment was well formed and smooth and not rough and vascular, which one expected to find in view of the suspicion of anterior placenta praevia. On incising the uterus, liquor drained out, and what presented at the uterine incision was foetal liver and gut. The diagnosis was then clear: this was a ruptured exomphalos, and what had been felt on vaginal examination was the two lobes of the foetal liver. The baby was lying obliquely, almost transversely, with the head under the right hypochondrium. It had visible deformities-a mild hydrocephalus and a marked scoliosis (Fig. 1).

On removing the baby, the placenta was found to be a second degree posterior placenta praevia which, of course, accounted for the antepartum haemorrhage. The Caesarean section was completed in the routine way and the patient made an uneventful convalescence.

The baby lived for 2-3 hr.

\section{Necropsy}

Cause of death: pulmonary atelectasis; exomphalos; prematurity.

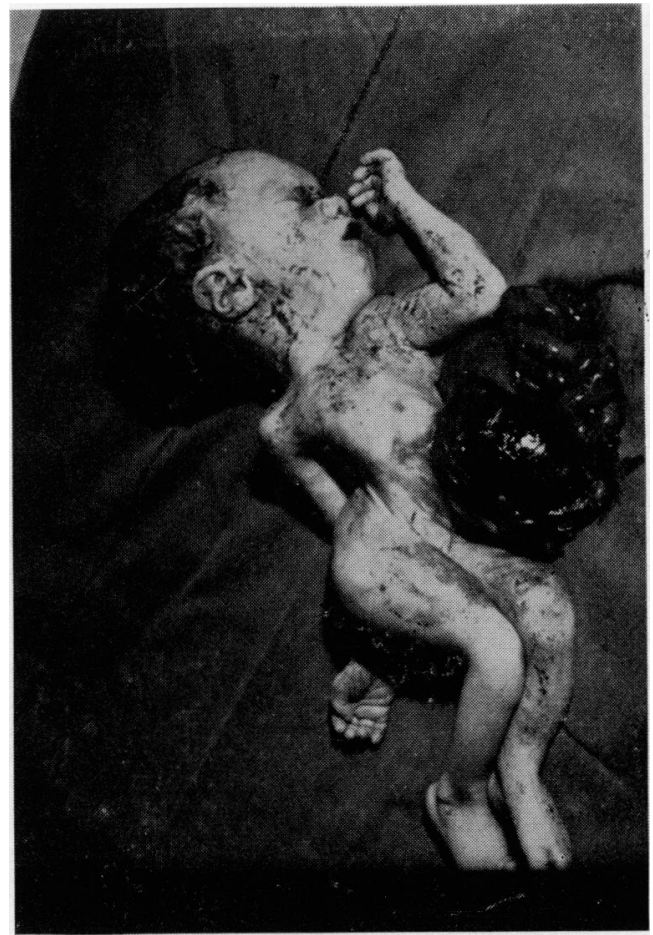

Fig. 1. The baby showing ruptured exomphalos and deformities.

External appearances and injuries: small female neonate, with exomphalos, liver, colon, spleen and small bowel presenting externally due to failure of development of abdominal wall and failure of return of foetal umbilical herniation.

Lungs: lungs collapsed, sink in water.

Peritoneum: wall deficient-exomphalos as previously described.

Intestines: normal, apart from exomphalos.

Liver: exomphalos; normal structure.

Spleen: exomphalos; structure normal.

Miscellaneous: anus and cardio-oesophageal junction patent. No ossification centre at lower end of femur. Marked lumbar scoliosis to the left.

\section{Discussion}

Could the diagnosis have been made earlier?

(1) Aird (1962) reporting a case of exomphalos presenting by the liver and intestines, quoted Fawcitt's (1953) points for X-ray diagnosis of such a foetus. He stresses the need for very high quality $\mathrm{X}$-ray, however, criteria being: (a) wide splaying of the lower ribs is present without the usual forward and inward curves, particularly of the eighth, ninth and tenth ribs; (b) the thoracic cage may make a pyramid from above downwards, resembling a 
church bell; and (c) the outline of the upper abdomen is protuberant, while the outline of the lower abdomen is normal.

(2) A soft tissue radiograph may have located the low lying placenta. Other methods of radiological diagnosis such as: (a) measuring the distance between the head and sacral promontory with the patient partially reclining and measuring the distance between the symphysis pubis and head in the erect position; and (b) cystography, would not have been any help as the head was not presenting. Amniography is not a procedure practised now.

(3) Ultrasonic scanning would have helped to locate the position of the foetal head and placenta.

Cases of omphalocele ruptured and unexplained have been reported on various occasions (Lothian, 1959; Williams et al., 1960; Leroux, 1961) but most of these have been diagnosed after delivery.

\section{If diagnosed earlier, would it have influenced manage- ment?}

In this instance, as there was bleeding due to the co-existing placenta praevia, one could argue that missing the diagnosis of exomphalos did not make a difference to the management, the management being immediate delivery, and this was by Caesarean section in the presence of only two fingers-breadth dilatation of the cervix.

Alternatively, if the condition of this malformed foetus had been diagnosed, one might have waited until the cervix was dilated to four fingers-breadth and then performed an internal podalic version thus keeping pressure on the placenta with a half breech until full dilatation, when vaginal delivery would have been possible, thus sparing this young unmarried girl a scar in her uterus.

\section{Acknowledgment}

I am deeply indebted to Mr I. A. Donaldson, the Consultant in charge of this case for his kind permission and encouragement to publish the case.

\section{References}

AIRD, D. (1962) An unusual presentation simulating placenta praevia. Brit. J. clin. Pract. 16, 773.

FAWCITT, J. (1953) The radiological diagnosis of exomphalos. (With a report of a case.) Brit. J. Radiol. 26, 210.

Leroux, P. (1961) A case of intrauterine rupture of amniotic hernia with total evisceration of the small intestine. Bull. Fed. Gynaec. Obstet. franc. 13, 208.

Lothian, W. (1959) A congenital amniotic hernia with the placenta adherent to the sac. Practitioner, 183, 75.

Williams, A. et al. (1960) Intrauterine rupture of omphalocele. Virginia Med. Monthly, 87, 94.

\title{
Intussusception following gastric surgery
}

\author{
JOHN MCL. SingLETON \\ M.B., F.R.C.S.* \\ Senior Surgical Registrar, \\ St George's Hospital, London, S.W.1
}

\begin{abstract}
ACUTE intussusception as a complication of gastric surgery is now a well-known though still uncommon event. That intussusception may occur in a chronic intermittent self-reducing form is a less widely recognized fact, and may be the cause of recurring gastro-intestinal symptoms. Eventually, the intussusception may fail to reduce and become incarcerated.
\end{abstract}

\section{Case report}

L.S., male aged 61 years, was admitted as an emergency. Following an early morning bout of sharp upper abdominal pain, he had been seized with severe continuous epigastric pain. He had vomited twice, the first occasion coffee-grounds, the second blood-stained mucus.

\footnotetext{
*Present address: Peter Bent Brigham Hospital, Boston, Massachusetts, U.S.A.
}

He had had a gastric operation 15 years previously in Australia and had had numerous episodes of pain, flatulence and distension since then, but one more severe episode 5 weeks previously while in Vienna had resulted in a barium meal being carried out - this was reported as normal.

Since a heart attack 3 years ago, he had been on anticoagulants but had not received any other regular drugs.

On examination, he was thin; there was an upper abdominal mass most prominent in the left hypochondrium which appeared to be as two unequal incomplete halves like an inverted cottage loaf. It was very tender and fixed. The remainder of the examination was unremarkable.

Investigations. $15.5 \mathrm{~g} / 100 \mathrm{ml}, \mathrm{PCV} 45 \%$, serum amylase 320 units (normal 0-200), Na, $131 \mathrm{mEq} / \mathrm{l}$, 